Open J. Math. Sci., Vol. 1(2017), No. 1, pp. 97-110

Website: https://pisrt.org/psr-press/journals/oms/

ISSN: 2523-0212 (Online) 2616-4906 (Print)

http://dx.doi.org/10.30538/oms2017.0010

\title{
OSTROWSKI TYPE FRACTIONAL INTEGRAL INEQUALITIES FOR $S$-GODUNOVA-LEVIN FUNCTIONS VIA KATUGAMPOLA FRACTIONAL INTEGRALS
}

\author{
GHULAM FARID ${ }^{1}$, UDITA N. KATUGAMPOLA, MUHAMMAD USMAN
}

\begin{abstract}
In this paper, we give some fractional integral inequalities of Ostrowski type for $s$-Godunova-Levin functions via Katugampola fractional integrals. We also deduce some known Ostrowski type fractional integral inequalities for Riemann-Liouville fractional integrals.
\end{abstract}

AMS Mathematics Subject Classification: 26A33, 26A51, 26D07, 26D10, $26 \mathrm{D} 15$.

Key words and phrases: Ostrowski inequality; fractional integral; s-GodunovaLevin functions.

\section{INTRODUCTION}

In 1938 Ostrowski [1] proved an inequality stated in the following result (see also [2, p.468]).

Theorem 1.1. Let $f: I \rightarrow \mathbb{R}$ where $I$ is interval in $\mathbb{R}$, be a mapping differentiable in $I^{\circ}$ the interior of $I$ and $a, b \in I^{\circ}, a<b$. If $\left|f^{\prime}(t)\right| \leq M$, for all $t \in[a, b]$, then we have

$$
\left|f(x)-\frac{1}{b-a} \int_{a}^{b} f(t) d t\right| \leq\left[\frac{1}{4}+\frac{\left(x-\frac{a+b}{2}\right)^{2}}{(b-a)^{2}}\right](b-a) M, x \in[a, b] .
$$

Ostrowski inequality gives bounds of integral average of a function $f$ over an interval $[a, b]$ to its value $f(x)$ at point $x \in[a, b]$. Ostrowski and Ostrowski type inequalities have great importance in numerical analysis as they provide the error bound of many quaderature rules [3]. Therefore in recent years, so many

Received 13 August 2017. Revised 28 November 2017.

1 Corresponding Author

(C) 2017 Ghulam Farid, Udita N. Katugampola, Muhammad Usman. This is an open access article distributed under the Creative Commons Attribution License, which permits unrestricted use, distribution, and reproduction in any medium, provided the original work is properly cited. 
such type of inequalities have been obtained and generalized (see $[4,5])$.

As fractional calculus is a generalization of classical calculus concerned with operations of integration and differentiation of fractional order so in this research article we will use Katugampola fractional integrals to generalize the Ostrowski type inequalities given in [4].

In [6] Laurent give definition of Riemann-Liouville fractional integrals.

Definition 1.2. [6] Let $f \in L_{1}[a, b]$. The Riemann-Liouville fractional integrals $J_{a+}^{\alpha} f$ and $J_{b-}^{\alpha} f$ of order $\alpha>0$ with $a \geq 0$ are defined by

$$
J_{a+}^{\alpha} f(x)=\frac{1}{\Gamma(\alpha)} \int_{a}^{x}(x-t)^{\alpha-1} f(t) d t, x>a
$$

and

$$
J_{b-}^{\alpha} f(x)=\frac{1}{\Gamma(\alpha)} \int_{x}^{b}(t-x)^{\alpha-1} f(t) d t, x<b,
$$

respectively, where $\Gamma(\alpha)=\int_{0}^{\infty} e^{-u} u^{\alpha-1} d u$. Here $\Gamma(\alpha+1)=\alpha \Gamma(\alpha)$, $J_{a+}^{0} f(x)=J_{b-}^{0} f(x)=f(x)$. In case of $\alpha=1$, the fractional integral reduces to the classical integral.

Definition 1.3. J. Hadamard introduced the Hadamard fractional integral in [7], and is given by

$$
I_{a^{+}}^{\alpha} f(x)=\frac{1}{\Gamma(\alpha)} \int_{a}^{x}\left(\log \frac{x}{\tau}\right)^{\alpha-1} f(\tau) \frac{d \tau}{\tau},
$$

for $\operatorname{Re}(\alpha)>0, x>a \geq 0$.

Recently Katugampola generalized Riemann-Liouville and Hadamard fractional integrals into a single form called Katugampola fractional integrals.

Definition 1.4. [8] Let $[a, b]$ be a finite interval in $\mathbb{R}$. Then Katugampola fractional integrals of order $\alpha>0$ for a real valued function $f$ are defined by

$$
{ }^{\rho} I_{a+}^{\alpha} f(x)=\frac{\rho^{1-\alpha}}{\Gamma(\alpha)} \int_{a}^{x} t^{\rho-1}\left(x^{\rho}-t^{\rho}\right)^{\alpha-1} f(t) d t
$$

and

$$
{ }^{\rho} I_{b-}^{\alpha} f(x)=\frac{\rho^{1-\alpha}}{\Gamma(\alpha)} \int_{x}^{b} t^{\rho-1}\left(t^{\rho}-x^{\rho}\right)^{\alpha-1} f(t) d t
$$

with $a<x<b$ and $\rho>0$.

Where $\Gamma(\alpha)$ is the Euler gamma function. For $\rho=1$, Katugampola fractional integrals give Riemann-Liouville fractional integrals, while $\rho \rightarrow 0^{+}$produces the Hadamard fractional integral. For its proof one can check [8].

The $\rho$-Gamma function [9] for any two positive numbers $x, y$ denoted by ${ }^{\rho} \Gamma(x, y)$, is defined by

$$
{ }^{\rho} \Gamma(\alpha)=\int_{0}^{\infty} e^{-t^{\rho}}\left(t^{\rho}\right)^{\alpha-\frac{1}{\rho}} d t .
$$


We can have the following relation

$$
{ }^{\rho} \beta(x, y)=\frac{{ }^{\rho} \Gamma(x)^{\rho} \Gamma(y)}{\rho \Gamma(x, y)} .
$$

Definition 1.5. [10] A non-negative function $f: I \rightarrow \mathbb{R}$ is said to be $p$-function, if for any two points $x, y \in I$ and $t \in[0,1]$

$$
f(t x+(1-t) y) \leq f(x)+f(y) .
$$

Definition 1.6. [11] A function $f: I \rightarrow \mathbb{R}$ is said to be Godunova-Levin function, if for any two points $x, y \in I$ and $t \in(0,1)$

$$
f(t x+(1-t) y) \leq \frac{f(x)}{t}+\frac{f(y)}{1-t} .
$$

Definition 1.7. [12] A function $f: I \rightarrow \mathbb{R}$ is said to be $s$-Godunova-Levin function of first kind, if $s \in(0,1]$, for all $x, y \in I$ and $t \in(0,1)$ then we have

$$
f(t x+(1-t) y) \leq \frac{f(x)}{t^{s}}+\frac{f(y)}{1-t^{s}} .
$$

Definition 1.8. [13] A function $f: I \rightarrow \mathbb{R}$ is said to be $s$-Godunova-Levin function of second kind, if $s \in[0,1]$, for all $x, y \in I$ and $t \in(0,1)$ then we have

$$
f(t x+(1-t) y) \leq \frac{f(x)}{t^{s}}+\frac{f(y)}{(1-t)^{s}} .
$$

We organize the paper in such a way that in the following section we prove some Ostrowski type fractional integral inequalities for $s$-Godunova-Levin functions of second kind via Katugampola fractional integrals. Also we will obtain some corollaries for $p$-functions and Godunova-Levin functions and deduce some known results of [4].

\section{Ostrowski type fractional integral inequalities for mappings whose derivatives are $s$-Godunova-Levin of second kind via Katugampola fractional integrals}

The following lemma (given and also proved in [9]) is very useful to obtain our results.

Lemma 2.1. Let $f:\left[a^{\rho}, b^{\rho}\right] \rightarrow \mathbb{R}$ be a differentiable mapping on $\left(a^{\rho}, b^{\rho}\right)$ with $a<b$ such that $f^{\prime} \in L_{1}[a, b]$, where $\rho>0$. Then we have the following equality

$$
\begin{aligned}
& \left(\frac{\left(x^{\rho}-a^{\rho}\right)^{\alpha}+\left(b^{\rho}-x^{\rho}\right)^{\alpha}}{b-a}\right) f\left(x^{\rho}\right)-\frac{(\alpha \rho+\rho-1) \Gamma(\alpha)}{\rho^{1-\alpha}(b-a)} \times \\
& {\left[{ }^{\rho} I_{x^{-}}^{\alpha} f\left(a^{\rho}\right)+{ }^{\rho} I_{x^{+}}^{\alpha} f\left(b^{\rho}\right)\right]} \\
& =\frac{\rho\left(x^{\rho}-a^{\rho}\right)^{\alpha+1}}{b-a} \int_{0}^{1} t^{\alpha \rho+\rho-1} f^{\prime}\left(t^{\rho} x^{\rho}+\left(1-t^{\rho}\right) a^{\rho}\right) d t \\
& -\frac{\rho\left(b^{\rho}-x^{\rho}\right)^{\alpha+1}}{b-a} \int_{0}^{1} t^{\alpha \rho+\rho-1} f^{\prime}\left(t^{\rho} x^{\rho}+\left(1-t^{\rho}\right) b^{\rho}\right) d t ; x \in[a, b] .
\end{aligned}
$$


Theorem 2.2. Let $f:\left[a^{\rho}, b^{\rho}\right] \rightarrow \mathbb{R}, a, b \geq 0, a<b$ be a differentiable function on $\left(a^{\rho}, b^{\rho}\right)$ and $f^{\prime} \in L_{1}[a, b]$. If $\left|f^{\prime}\right|$ is s-Godunova-Levin function of second kind and $\left|f^{\prime}\left(x^{\rho}\right)\right| \leq M, x \in[a, b]$, then the following inequality holds

$$
\begin{aligned}
& \mid\left(\frac{\left(x^{\rho}-a^{\rho}\right)^{\alpha}+\left(b^{\rho}-x^{\rho}\right)^{\alpha}}{b-a}\right) f\left(x^{\rho}\right)-\frac{(\alpha \rho+\rho-1) \Gamma(\alpha)}{\rho^{1-\alpha}(b-a)} \times \\
& {\left[{ }^{\rho} I_{x^{-}}^{\alpha} f\left(a^{\rho}\right)+{ }^{\rho} I_{x^{+}}^{\alpha} f\left(b^{\rho}\right)\right] \mid \leq M\left[\frac{\left(x^{\rho}-a^{\rho}\right)^{\alpha+1}+\left(b^{\rho}-x^{\rho}\right)^{\alpha+1}}{b-a}\right] \times} \\
& {\left[\frac{1}{\alpha+1-s}+\frac{{ }^{\rho} \Gamma(\alpha+1){ }^{\rho} \Gamma(1-s)}{{ }^{\rho} \Gamma(\alpha+2-s)}\right] ; x \in[a, b] .}
\end{aligned}
$$

Proof. Using Lemma 2.1 and the fact that $\left|f^{\prime}\right|$ is $s$-Godunova-Levin function of second kind, we have

$$
\begin{aligned}
& \mid\left(\frac{\left(x^{\rho}-a^{\rho}\right)^{\alpha}+\left(b^{\rho}-x^{\rho}\right)^{\alpha}}{b-a}\right) f\left(x^{\rho}\right)-\frac{(\alpha \rho+\rho-1) \Gamma(\alpha)}{\rho^{1-\alpha}(b-a)} \times \\
& {\left[{ }^{\rho} I_{x^{-}}^{\alpha} f\left(a^{\rho}\right)+{ }^{\rho} I_{x^{+}}^{\alpha} f\left(b^{\rho}\right)\right]} \\
& \leq \frac{\rho\left(x^{\rho}-a^{\rho}\right)^{\alpha+1}}{b-a} \int_{0}^{1} t^{\alpha \rho+\rho-1}\left|f^{\prime}\left(t^{\rho} x^{\rho}+\left(1-t^{\rho}\right) a^{\rho}\right)\right| d t \\
& +\frac{\rho\left(b^{\rho}-x^{\rho}\right)^{\alpha+1}}{b-a} \int_{0}^{1} t^{\alpha \rho+\rho-1}\left|f^{\prime}\left(t^{\rho} x^{\rho}+\left(1-t^{\rho}\right) b^{\rho}\right)\right| d t \\
& \leq \frac{\rho\left(x^{\rho}-a^{\rho}\right)^{\alpha+1}}{b-a} \int_{0}^{1}\left[\frac{t^{\alpha \rho+\rho-1}}{\left(t^{\rho}\right)^{s}}\left|f^{\prime}\left(x^{\rho}\right)\right|+\frac{t^{\alpha \rho+\rho-1}}{\left(1-t^{\rho}\right)^{s}}\left|f^{\prime}\left(a^{\rho}\right)\right|\right] d t \\
& +\frac{\rho\left(b^{\rho}-x^{\rho}\right)^{\alpha+1}}{b-a} \int_{0}^{1}\left[\frac{t^{\alpha \rho+\rho-1}}{\left(t^{\rho}\right)^{s}}\left|f^{\prime}\left(x^{\rho}\right)\right|+\frac{t^{\alpha \rho+\rho-1}}{\left(1-t^{\rho}\right)^{s}}\left|f^{\prime}\left(b^{\rho}\right)\right|\right] d t \\
& \leq \frac{M \rho\left(x^{\rho}-a^{\rho}\right)^{\alpha+1}}{b-a} \int_{0}^{1}\left[\frac{t^{\alpha \rho+\rho-1}}{\left(t^{\rho}\right)^{s}}+\frac{t^{\alpha \rho+\rho-1}}{\left(1-t^{\rho}\right)^{s}}\right] d t \\
& +\frac{M \rho\left(b^{\rho}-x^{\rho}\right)^{\alpha+1}}{b-a} \int_{0}^{1}\left[\frac{t^{\alpha \rho+\rho-1}}{\left(t^{\rho}\right)^{s}}+\frac{t^{\alpha \rho+\rho-1}}{\left(1-t^{\rho}\right)^{s}}\right] d t \\
& =M \rho\left[\frac{\left(x^{\rho}-a^{\rho}\right)^{\alpha+1}+\left(b^{\rho}-x^{\rho}\right)^{\alpha+1}}{2(b-a)}\right] \times \\
& \int_{0}^{1}\left[t^{\alpha \rho-\rho s+\rho-1}+t^{\alpha \rho+\rho-1}\left(1-t^{\rho}\right)^{-s}\right] d t . \\
& =M \rho\left[\frac{\left(x^{\rho}-a^{\rho}\right)^{\alpha+1}+\left(b^{\rho}-x^{\rho}\right)^{\alpha+1}}{2(b-a)}\right] \times \\
& {\left[\frac{1}{\rho(\alpha+1-s)}+\frac{{ }^{\rho} \Gamma(\alpha+1)^{\rho} \Gamma(1-s)}{\rho^{\rho} \Gamma(\alpha+2-s)}\right]} \\
& =M\left[\frac{\left(x^{\rho}-a^{\rho}\right)^{\alpha+1}+\left(b^{\rho}-x^{\rho}\right)^{\alpha+1}}{b-a}\right] \times
\end{aligned}
$$




$$
\left[\frac{1}{\alpha+1-s}+\frac{{ }^{\rho} \Gamma(\alpha+1)^{\rho} \Gamma(1-s)}{\rho \Gamma(\alpha+2-s)}\right] .
$$

Here we use (1). The proof is completed.

Remark 2.3. (i) If we put $\rho=1$ in (3), then we get [4, Theorem 3.1].

(ii) If we put $\rho=1$ and $\alpha=1$ in (3), then we get [4, Corollary 3.1].

Corollary 2.4. In Theorem 2.2, if we take $s=0$, which means that $\left|f^{\prime}\right|$ is p-function, then (3) becomes the following inequality

$$
\begin{aligned}
& \mid\left(\frac{\left(x^{\rho}-a^{\rho}\right)^{\alpha}+\left(b^{\rho}-x^{\rho}\right)^{\alpha}}{b-a}\right) f\left(x^{\rho}\right)-\frac{(\alpha \rho+\rho-1) \Gamma(\alpha)}{\rho^{1-\alpha}(b-a)} \times \\
& {\left[{ }^{\rho} I_{x^{-}}^{\alpha} f\left(a^{\rho}\right)+{ }^{\rho} I_{x^{+}}^{\alpha} f\left(b^{\rho}\right)\right] \mid} \\
& \leq \frac{2 M}{\alpha+1}\left[\frac{\left(x^{\rho}-a^{\rho}\right)^{\alpha+1}+\left(b^{\rho}-x^{\rho}\right)^{\alpha+1}}{b-a}\right] ; x \in[a, b] .
\end{aligned}
$$

Corollary 2.5. In Theorem 2.2, if we take $s=1$, which means that $\left|f^{\prime}\right|$ is Godunova-Levin function, then (3) becomes the following inequality

$$
\begin{aligned}
& \mid\left(\frac{\left(x^{\rho}-a^{\rho}\right)^{\alpha}+\left(b^{\rho}-x^{\rho}\right)^{\alpha}}{b-a}\right) f\left(x^{\rho}\right)-\frac{(\alpha \rho+\rho-1) \Gamma(\alpha)}{\rho^{1-\alpha}(b-a)} \times \\
& {\left[{ }^{\rho} I_{x^{-}}^{\alpha} f\left(a^{\rho}\right)+{ }^{\rho} I_{x^{+}}^{\alpha} f\left(b^{\rho}\right)\right] \mid} \\
& \leq \frac{M(\alpha+1)}{\alpha}\left[\frac{\left(x^{\rho}-a^{\rho}\right)^{\alpha+1}+\left(b^{\rho}-x^{\rho}\right)^{\alpha+1}}{b-a}\right] ; x \in[a, b] .
\end{aligned}
$$

Theorem 2.6. Let $f:\left[a^{\rho}, b^{\rho}\right] \rightarrow \mathbb{R}, a, b \geq 0, a<b$ be a differentiable function on $\left(a^{\rho}, b^{\rho}\right)$ and $f^{\prime} \in L_{1}[a, b]$. If $\left|f^{\prime}\right|^{q}$, is s-Godunova-Levin function of second kind and $\left|f^{\prime}\left(x^{\rho}\right)\right| \leq M, x \in[a, b]$ then the following inequality for Katugampola fractional integrals holds

$$
\begin{aligned}
& \mid\left(\frac{\left(x^{\rho}-a^{\rho}\right)^{\alpha}+\left(b^{\rho}-x^{\rho}\right)^{\alpha}}{b-a}\right) f\left(x^{\rho}\right)-\frac{(\alpha \rho+\rho-1) \Gamma(\alpha)}{\rho^{1-\alpha}(b-a)} \times \\
& {\left[{ }^{\rho} I_{x^{-}}^{\alpha} f\left(a^{\rho}\right)+{ }^{\rho} I_{x^{+}}^{\alpha} f\left(b^{\rho}\right)\right] \mid} \\
& \leq M \rho\left[\frac{\left(x^{\rho}-a^{\rho}\right)^{\alpha+1}+\left(b^{\rho}-x^{\rho}\right)^{\alpha+1}}{(b-a)(1+p(\alpha \rho+\rho-1))^{\frac{1}{p}}}\right]\left[\frac{1}{1-\rho s}\right]^{\frac{1}{q}} ; x \in[a, b],
\end{aligned}
$$

with $\frac{1}{p}+\frac{1}{q}=1$ where $q>1$.

Proof. Using Lemma 2.1 and then Holder's inequality, we have

$$
\mid\left(\frac{\left(x^{\rho}-a^{\rho}\right)^{\alpha}+\left(b^{\rho}-x^{\rho}\right)^{\alpha}}{b-a}\right) f\left(x^{\rho}\right)-\frac{(\alpha \rho+\rho-1) \Gamma(\alpha)}{\rho^{1-\alpha}(b-a)} \times
$$




$$
\begin{aligned}
& {\left[{ }^{\rho} I_{x^{-}}^{\alpha} f\left(a^{\rho}\right)+{ }^{\rho} I_{x^{+}}^{\alpha} f\left(b^{\rho}\right)\right] \mid} \\
& \leq \frac{\rho\left(x^{\rho}-a^{\rho}\right)^{\alpha+1}}{b-a} \int_{0}^{1} t^{\alpha \rho+\rho-1}\left|f^{\prime}\left(t^{\rho} x^{\rho}+\left(1-t^{\rho}\right) a^{\rho}\right)\right| d t \\
& +\frac{\rho\left(b^{\rho}-x^{\rho}\right)^{\alpha+1}}{b-a} \int_{0}^{1} t^{\alpha \rho+\rho-1}\left|f^{\prime}\left(t^{\rho} x^{\rho}+\left(1-t^{\rho}\right) b^{\rho}\right)\right| d t \\
& \leq \frac{\rho\left(x^{\rho}-a^{\rho}\right)^{\alpha+1}}{b-a}\left(\int_{0}^{1} t^{p(\alpha \rho+\rho-1)} d t\right)^{\frac{1}{p}}\left(\int_{0}^{1}\left|f^{\prime}\left(t^{\rho} x^{\rho}+\left(1-t^{\rho}\right) a^{\rho}\right)\right|^{q} d t\right)^{\frac{1}{q}} \\
& +\frac{\rho\left(b^{\rho}-x^{\rho}\right)^{\alpha+1}}{b-a}\left(\int_{0}^{1} t^{p(\alpha \rho+\rho-1)} d t\right)^{\frac{1}{p}}\left(\int_{0}^{1}\left|f^{\prime}\left(t^{\rho} x^{\rho}+\left(1-t^{\rho}\right) b^{\rho}\right)\right|^{q} d t\right)^{\frac{1}{q}} .
\end{aligned}
$$

Since $\left|f^{\prime}\right|^{q}$ is $s$-Godunova-Levin function of second kind and $\left|f^{\prime}\left(x^{\rho}\right)\right| \leq M$, we get

$$
\begin{aligned}
& \int_{0}^{1}\left|f^{\prime}\left(t^{\rho} x^{\rho}+\left(1-t^{\rho}\right) a^{\rho}\right)\right|^{q} d t \times \\
& \leq \int_{0}^{1}\left[\frac{1}{\left(t^{\rho}\right)^{s}}\left|f^{\prime}\left(x^{\rho}\right)\right|^{q}+\frac{1}{\left(1-t^{\rho}\right)^{s}}\left|f^{\prime}\left(a^{\rho}\right)\right|^{q}\right] d t \\
& \leq M^{q} \int_{0}^{1}\left[\frac{1}{\left(t^{\rho}\right)^{s}}+\frac{1}{\left(1-t^{\rho}\right)^{s}}\right] d t=\frac{1}{1-\rho s}
\end{aligned}
$$

similarly

$$
\int_{0}^{1}\left|f^{\prime}\left(t^{\rho} x^{\rho}+\left(1-t^{\rho}\right) b^{\rho}\right)\right|^{q} d t \leq \frac{1}{1-\rho s} .
$$

We also have

$$
\int_{0}^{1} t^{p(\alpha \rho+\rho-1)} d t=\frac{1}{1+p(\alpha \rho+\rho-1)} .
$$

Using (6), (7) and (8) in (5) we can get (4).

Remark 2.7. (i) If we put $\rho=1$ in (4), then we get [4, Theorem 3.2].

(ii) If we put $\rho=1$ and $\alpha=1$ in (4), then we get [4, Corollary 3.2].

Corollary 2.8. In Theorem 2.6, if we take $s=0$, which means that $\left|f^{\prime}\right|$ is p-function, then (4) becomes the following inequality

$$
\begin{aligned}
& \mid\left(\frac{\left(x^{\rho}-a^{\rho}\right)^{\alpha}+\left(b^{\rho}-x^{\rho}\right)^{\alpha}}{b-a}\right) f\left(x^{\rho}\right)-\frac{(\alpha \rho+\rho-1) \Gamma(\alpha)}{\rho^{1-\alpha}(b-a)} \times \\
& {\left[{ }^{\rho} I_{x^{-}}^{\alpha} f\left(a^{\rho}\right)+{ }^{\rho} I_{x^{+}}^{\alpha} f\left(b^{\rho}\right)\right] \mid} \\
& \leq \frac{M}{(1+p(\alpha \rho+\rho-1))^{\frac{1}{p}}}\left[\frac{\left(x^{\rho}-a^{\rho}\right)^{\alpha+1}+\left(b^{\rho}-x^{\rho}\right)^{\alpha+1}}{b-a}\right] ; x \in[a, b] .
\end{aligned}
$$


Corollary 2.9. In Theorem, if we take $s=1$, which means that $\left|f^{\prime}\right|$ is Godunova-Levin function, then (4) becomes the following inequality

$$
\begin{aligned}
& \mid\left(\frac{\left(x^{\rho}-a^{\rho}\right)^{\alpha}+\left(b^{\rho}-x^{\rho}\right)^{\alpha}}{b-a}\right) f\left(x^{\rho}\right)-\frac{(\alpha \rho+\rho-1) \Gamma(\alpha)}{\rho^{1-\alpha}(b-a)} \times \\
& {\left[{ }^{\rho} I_{x^{-}}^{\alpha} f\left(a^{\rho}\right)+{ }^{\rho} I_{x^{+}}^{\alpha} f\left(b^{\rho}\right)\right] \mid \leq \frac{M \rho}{(1+p(\alpha \rho+\rho-1))^{\frac{1}{p}}} \times} \\
& {\left[\frac{\left(x^{\rho}-a^{\rho}\right)^{\alpha+1}+\left(b^{\rho}-x^{\rho}\right)^{\alpha+1}}{b-a}\right]\left[\frac{1+\alpha}{\alpha \rho}\right]^{\frac{1}{q}} ; x \in[a, b] .}
\end{aligned}
$$

Theorem 2.10. Let $f:\left[a^{\rho}, b^{\rho}\right] \rightarrow \mathbb{R}, a, b \geq 0, a<b$ be a differentiable function on $\left(a^{\rho}, b^{\rho}\right)$ and $f^{\prime} \in L_{1}[a, b]$. If $\left|f^{\prime}\right|^{q}$ is s-Godunova-Levin function of second kind and $\left|f^{\prime}\left(x^{\rho}\right)\right| \leq M, x \in[a, b], q \geq 1$, then the following inequality for Katugampola fractional integrals holds

$$
\begin{aligned}
& \mid\left(\frac{\left(x^{\rho}-a^{\rho}\right)^{\alpha}+\left(b^{\rho}-x^{\rho}\right)^{\alpha}}{b-a}\right) f\left(x^{\rho}\right)-\frac{(\alpha \rho+\rho-1) \Gamma(\alpha)}{\rho^{1-\alpha}(b-a)} \times \\
& {\left[{ }^{\rho} I_{x^{-}}^{\alpha} f\left(a^{\rho}\right)+{ }^{\rho} I_{x^{+}}^{\alpha} f\left(b^{\rho}\right)\right] \mid} \\
& \leq \frac{M \rho}{(\alpha \rho+\rho)^{1-\frac{1}{q}}}\left[\frac{\left(x^{\rho}-a^{\rho}\right)^{\alpha+1}+\left(b^{\rho}-x^{\rho}\right)^{\alpha+1}}{b-a}\right] \times \\
& \left(\frac{1}{\rho(\alpha-s+1)}+\frac{{ }^{\rho} \Gamma(\alpha+1)^{\rho} \Gamma(1-s)}{\rho^{\rho} \Gamma(\alpha-s+2)}\right)^{\frac{1}{q}} ; x \in[a, b] .
\end{aligned}
$$

Proof. Using Lemma 2.1 and power mean inequality, we have

$$
\begin{aligned}
& \mid\left(\frac{\left(x^{\rho}-a^{\rho}\right)^{\alpha}+\left(b^{\rho}-x^{\rho}\right)^{\alpha}}{b-a}\right) f\left(x^{\rho}\right)-\frac{(\alpha \rho+\rho-1) \Gamma(\alpha)}{\rho^{1-\alpha}(b-a)} \times \\
& {\left[{ }^{\rho} I_{x^{-}}^{\alpha} f\left(a^{\rho}\right)+{ }^{\rho} I_{x^{+}}^{\alpha} f\left(b^{\rho}\right)\right] \mid} \\
& \leq \frac{\rho\left(x^{\rho}-a^{\rho}\right)^{\alpha+1}}{b-a} \int_{0}^{1} t^{\alpha \rho+\rho-1}\left|f^{\prime}\left(t^{\rho} x^{\rho}+\left(1-t^{\rho}\right) a^{\rho}\right)\right| d t \\
& +\frac{\rho\left(b^{\rho}-x^{\rho}\right)^{\alpha+1}}{b-a} \int_{0}^{1} t^{\alpha \rho+\rho-1}\left|f^{\prime}\left(t^{\rho} x^{\rho}+\left(1-t^{\rho}\right) b^{\rho}\right)\right| d t \\
& \leq \frac{\rho\left(x^{\rho}-a^{\rho}\right)^{\alpha+1}}{b-a}\left(\int_{0}^{1} t^{\alpha \rho+\rho-1} d t\right)^{1-\frac{1}{q}} \times \\
& \left(\int_{0}^{1} t^{\alpha \rho+\rho-1}\left|f^{\prime}\left(t^{\rho} x^{\rho}+\left(1-t^{\rho}\right) a^{\rho}\right)\right|^{q} d t\right)^{\frac{1}{q}} \\
& +\frac{\rho\left(b^{\rho}-x^{\rho}\right)^{\alpha+1}}{b-a}\left(\int_{0}^{1} t^{\alpha \rho+\rho-1} d t\right)^{1-\frac{1}{q}} \times
\end{aligned}
$$




$$
\left(\int_{0}^{1} t^{\alpha \rho+\rho-1}\left|f^{\prime}\left(t^{\rho} x^{\rho}+\left(1-t^{\rho}\right) b^{\rho}\right)\right|^{q} d t\right)^{\frac{1}{q}}
$$

Since $\left|f^{\prime}\right|^{q}$ is $s$-Godunova-Levin function of second kind and $\left|f^{\prime}\left(x^{\rho}\right)\right| \leq M$, we get

$$
\begin{aligned}
& \int_{0}^{1} t^{\alpha \rho+\rho-1}\left|f^{\prime}\left(t^{\rho} x^{\rho}+\left(1-t^{\rho}\right) a^{\rho}\right)\right|^{q} d t \\
& \leq \int_{0}^{1}\left[\frac{t^{\alpha \rho+\rho-1}}{\left(t^{\rho}\right)^{s}}\left|f^{\prime}\left(x^{\rho}\right)\right|^{q}+\frac{t^{\alpha \rho+\rho-1}}{\left(1-t^{\rho}\right)^{s}}\left|f^{\prime}\left(a^{\rho}\right)\right|^{q}\right] d t \\
& \leq M^{q} \int_{0}^{1}\left[\frac{t^{\alpha \rho+\rho-1}}{\left(t^{\rho}\right)^{s}}+\frac{t^{\alpha \rho+\rho-1}}{\left(1-t^{\rho}\right)^{s}}\right] d t \times \\
& =M^{q}\left[\frac{1}{\rho(\alpha-s+1)}+\frac{{ }^{\rho} \Gamma(\alpha+1)^{\rho} \Gamma(1-s)}{\rho^{\rho} \Gamma(\alpha-s+2)}\right]
\end{aligned}
$$

similarly

$$
\begin{aligned}
& \int_{0}^{1} t^{\alpha \rho+\rho-1}\left|f^{\prime}\left(t^{\rho} x^{\rho}+\left(1-t^{\rho}\right) b^{\rho}\right)\right|^{q} d t \times \\
& \leq M^{q}\left[\frac{1}{\rho(\alpha-s+1)}+\frac{{ }^{\rho} \Gamma(\alpha+1)^{\rho} \Gamma(1-s)}{\rho^{\rho} \Gamma(\alpha-s+2)}\right] .
\end{aligned}
$$

Using (11) and (12) in (10) we can attain (9).

Remark 2.11. (i) If we put $\rho=1$ in (9), then we get [4, Theorem 3.3].

(ii) If we put $\rho=1$ and $\alpha=1$ in (9), then we get [4, Corollary 3.3].

Corollary 2.12. In Theorem 2.10, if we take $s=0$, which means that $\left|f^{\prime}\right|$ is p-function, then (9) becomes the following inequality

$$
\begin{aligned}
& \mid\left(\frac{\left(x^{\rho}-a^{\rho}\right)^{\alpha}+\left(b^{\rho}-x^{\rho}\right)^{\alpha}}{b-a}\right) f\left(x^{\rho}\right)-\frac{(\alpha \rho+\rho-1) \Gamma(\alpha)}{\rho^{1-\alpha}(b-a)} \times \\
& {\left[{ }^{\rho} I_{x^{-}}^{\alpha} f\left(a^{\rho}\right)+{ }^{\rho} I_{x^{+}}^{\alpha} f\left(b^{\rho}\right)\right] \mid} \\
& \leq \frac{M \rho}{(\alpha \rho+\rho)^{1-\frac{1}{q}}}\left[\frac{\left(x^{\rho}-a^{\rho}\right)^{\alpha+1}+\left(b^{\rho}-x^{\rho}\right)^{\alpha+1}}{b-a}\right]\left[\frac{2}{\rho(\alpha+1)}\right]^{\frac{1}{q}} ; x \in[a, b] .
\end{aligned}
$$

Corollary 2.13. In Theorem 2.10, if we take $s=1$, which means that $\left|f^{\prime}\right|$ is Godunova-Levin function, then (9) becomes the following inequality

$$
\begin{aligned}
& \mid\left(\frac{\left(x^{\rho}-a^{\rho}\right)^{\alpha}+\left(b^{\rho}-x^{\rho}\right)^{\alpha}}{b-a}\right) f\left(x^{\rho}\right)-\frac{(\alpha \rho+\rho-1) \Gamma(\alpha)}{\rho^{1-\alpha}(b-a)} \times \\
& {\left[{ }^{\rho} I_{x^{-}}^{\alpha} f\left(a^{\rho}\right)+{ }^{\rho} I_{x^{+}}^{\alpha} f\left(b^{\rho}\right)\right] \mid} \\
& \leq \frac{M \rho}{(\alpha \rho+\rho)^{1-\frac{1}{q}}}\left[\frac{\left(x^{\rho}-a^{\rho}\right)^{\alpha+1}+\left(b^{\rho}-x^{\rho}\right)^{\alpha+1}}{b-a}\right]\left[\frac{1+\alpha}{\alpha \rho}\right]^{\frac{1}{q}} ; x \in[a, b] .
\end{aligned}
$$


We use the following lemma to establish some new results. Its proof is similar to Lemma 2.1.

Lemma 2.14. Let $f:\left[a^{\rho}, b^{\rho}\right] \rightarrow \mathbb{R}$ be a differentiable mapping on $\left(a^{\rho}, b^{\rho}\right)$ with $a^{\rho}<b^{\rho}$ such that $f^{\prime} \in L_{1}\left[a^{\rho}, b^{\rho}\right]$, where $\rho>0$. Then we have the following equality

$$
\begin{aligned}
& f\left(x^{\rho}\right)-\frac{(\alpha \rho+\rho-1) \Gamma(\alpha)}{\rho^{1-\alpha}}\left[\frac{{ }^{\rho} I_{x^{-}}^{\alpha} f\left(a^{\rho}\right)}{2\left(x^{\rho}-a^{\rho}\right)^{\alpha}}+\frac{{ }^{\rho} I_{x^{+}}^{\alpha} f\left(b^{\rho}\right)}{2\left(b^{\rho}-x^{\rho}\right)^{\alpha}}\right] \\
& =\frac{\rho\left(x^{\rho}-a^{\rho}\right)}{2} \int_{0}^{1} t^{\alpha \rho+\rho-1} f^{\prime}\left(t^{\rho} x^{\rho}+\left(1-t^{\rho}\right) a^{\rho}\right) d t \\
& -\frac{\left(b^{\rho}-x^{\rho}\right)}{2} \int_{0}^{1} t^{\alpha \rho+\rho-1} f^{\prime}\left(t^{\rho} x^{\rho}+\left(1-t^{\rho}\right) b^{\rho}\right) d t ; x \in[a, b] .
\end{aligned}
$$

Theorem 2.15. Let $f:\left[a^{\rho}, b^{\rho}\right] \rightarrow \mathbb{R}, a, b \geq 0, a<b$ be a differentiable function on $\left(a^{\rho}, b^{\rho}\right)$ and $f^{\prime} \in L_{1}[a, b]$. If $\left|f^{\prime}\right|$ is s-Godunova-Levin function of second kind and $\left|f^{\prime}\left(x^{\rho}\right)\right| \leq M, x \in[a, b]$, then the following inequality holds

$$
\begin{aligned}
& \left|f\left(x^{\rho}\right)-\frac{(\alpha \rho+\rho-1) \Gamma(\alpha)}{\rho^{1-\alpha}}\left[\frac{\rho^{\rho} I_{x^{-}}^{\alpha} f\left(a^{\rho}\right)}{2\left(x^{\rho}-a^{\rho}\right)^{\alpha}}+\frac{\rho^{\rho} I_{x^{+}}^{\alpha} f\left(b^{\rho}\right)}{2\left(b^{\rho}-x^{\rho}\right)^{\alpha}}\right]\right| \\
& \leq \frac{M\left(b^{\rho}-a^{\rho}\right)}{2}\left[\frac{1}{\alpha-s+1}+\frac{{ }^{\rho} \Gamma(\alpha+1)^{\rho} \Gamma(1-s)}{\rho \Gamma(\alpha-s+2)}\right] ; x \in[a, b] .
\end{aligned}
$$

Proof. Using Corollary 2.8 and $s$-Godunova-Levin function of second kind of $\left|f^{\prime}\right|$ we proceed as follows

$$
\begin{aligned}
& \left|f\left(x^{\rho}\right)-\frac{(\alpha \rho+\rho-1) \Gamma(\alpha)}{\rho^{1-\alpha}}\left[\frac{{ }^{\rho} I_{x^{-}}^{\alpha} f\left(a^{\rho}\right)}{2\left(x^{\rho}-a^{\rho}\right)^{\alpha}}+\frac{\rho I_{x^{+}}^{\alpha} f\left(b^{\rho}\right)}{2\left(b^{\rho}-x^{\rho}\right)^{\alpha}}\right]\right| \\
& \leq \frac{\rho\left(x^{\rho}-a^{\rho}\right)}{2} \int_{0}^{1} t^{\alpha \rho+\rho-1}\left|f^{\prime}\left(t^{\rho} x^{\rho}+\left(1-t^{\rho}\right) a^{\rho}\right)\right| d t \\
& +\frac{\rho\left(b^{\rho}-x^{\rho}\right)}{2} \int_{0}^{1} t^{\alpha \rho+\rho-1}\left|f^{\prime}\left(t^{\rho} x^{\rho}+\left(1-t^{\rho}\right) b^{\rho}\right)\right| d t \\
& \leq \frac{\rho\left(x^{\rho}-a^{\rho}\right)}{2} \int_{0}^{1}\left[\frac{t^{\alpha \rho+\rho-1}}{\left(t^{\rho}\right)^{s}}\left|f^{\prime}\left(x^{\rho}\right)\right|+\frac{t^{\alpha \rho+\rho-1}}{\left(1-t^{\rho}\right)^{s}}\left|f^{\prime}\left(a^{\rho}\right)\right|\right] d t \\
& +\frac{\rho\left(b^{\rho}-x^{\rho}\right)}{2} \int_{0}^{1}\left[\frac{t^{\alpha \rho+\rho-1}}{\left(t^{\rho}\right)^{s}}\left|f^{\prime}\left(x^{\rho}\right)\right|+\frac{t^{\alpha \rho+\rho-1}}{\left(1-t^{\rho}\right)^{s}}\left|f^{\prime}\left(b^{\rho}\right)\right|\right] d t \\
& \leq \frac{M \rho\left(x^{\rho}-a^{\rho}\right)}{2} \int_{0}^{1}\left[\frac{t^{\alpha \rho+\rho-1}}{\left(t^{\rho}\right)^{s}}+\frac{t^{\alpha \rho+\rho-1}}{\left(1-t^{\rho}\right)^{s}}\right] d t \\
& +\frac{M \rho\left(b^{\rho}-x^{\rho}\right)}{2} \int_{0}^{1}\left[\frac{t^{\alpha \rho+\rho-1}}{\left(t^{\rho}\right)^{s}}+\frac{t^{\alpha \rho+\rho-1}}{\left(1-t^{\rho}\right)^{s}}\right] d t \\
& =M \rho\left[\frac{\left(x^{\rho}-a^{\rho}\right)+\left(b^{\rho}-x^{\rho}\right)}{2}\right] \int_{0}^{1}\left[t^{\alpha \rho-\rho s+\rho-1}+t^{\alpha \rho+\rho-1}\left(1-t^{\rho}\right)^{-s}\right] d t .
\end{aligned}
$$




$$
\begin{aligned}
& =M \rho\left[\frac{\left(x^{\rho}-a^{\rho}\right)+\left(b^{\rho}-x^{\rho}\right)}{2}\right]\left[\frac{1}{\rho(\alpha-s+1)}+\frac{{ }^{\rho} \Gamma(\alpha+1)^{\rho} \Gamma(1-s)}{\rho^{\rho} \Gamma(\alpha-s+2)}\right] \\
& =\frac{M\left(b^{\rho}-a^{\rho}\right)}{2}\left[\frac{1}{\alpha-s+1}+\frac{{ }^{\rho} \Gamma(\alpha+1)^{\rho} \Gamma(1-s)}{\rho \Gamma(\alpha-s+2)}\right] .
\end{aligned}
$$

Here we use (1). The proof is completed.

Corollary 2.16. In Theorem 2.15, if we take $s=0$, which means that $\left|f^{\prime}\right|$ is p-function, then (14) becomes the following inequality

$$
\begin{aligned}
& \left|f\left(x^{\rho}\right)-\frac{(\alpha \rho+\rho-1) \Gamma(\alpha)}{\rho^{1-\alpha}}\left[\frac{{ }^{\rho} I_{x^{-}}^{\alpha} f\left(a^{\rho}\right)}{2\left(x^{\rho}-a^{\rho}\right)^{\alpha}}+\frac{{ }^{\rho} I_{x^{+}}^{\alpha} f\left(b^{\rho}\right)}{2\left(b^{\rho}-x^{\rho}\right)^{\alpha}}\right]\right| \\
& \leq \frac{M\left(b^{\rho}-a^{\rho}\right)}{\alpha+1} ; x \in[a, b] .
\end{aligned}
$$

Corollary 2.17. In Theorem 2.15, if we take $s=1$, which means that $\left|f^{\prime}\right|$ is Godunova-Levin function, then (14) becomes the following inequality

$$
\begin{aligned}
& \left|f\left(x^{\rho}\right)-\frac{(\alpha \rho+\rho-1) \Gamma(\alpha)}{\rho^{1-\alpha}}\left[\frac{{ }^{\rho} I_{x^{-}}^{\alpha} f\left(a^{\rho}\right)}{2\left(x^{\rho}-a^{\rho}\right)^{\alpha}}+\frac{{ }^{\rho} I_{x^{+}}^{\alpha} f\left(b^{\rho}\right)}{2\left(b^{\rho}-x^{\rho}\right)^{\alpha}}\right]\right| \\
& \leq \frac{M(\alpha+1)\left(b^{\rho}-a^{\rho}\right)}{2 \alpha} ; x \in[a, b] .
\end{aligned}
$$

Theorem 2.18. Let $f:\left[a^{\rho}, b^{\rho}\right] \rightarrow \mathbb{R}, a, b \geq 0, a<b$ be a differentiable function on $\left(a^{\rho}, b^{\rho}\right)$ and $f^{\prime} \in L_{1}[a, b]$. If $\left|f^{\prime}\right|^{q}$, is s-Godunova-Levin function of second kind and $\left|f^{\prime}\left(x^{\rho}\right)\right| \leq M, x \in[a, b]$ then the following inequality for Katugampola fractional integrals holds

$$
\begin{aligned}
& \left|f\left(x^{\rho}\right)-\frac{(\alpha \rho+\rho-1) \Gamma(\alpha)}{\rho^{1-\alpha}}\left[\frac{{ }^{\rho} I_{x^{-}}^{\alpha} f\left(a^{\rho}\right)}{2\left(x^{\rho}-a^{\rho}\right)^{\alpha}}+\frac{{ }^{\rho} I_{x^{+}}^{\alpha} f\left(b^{\rho}\right)}{2\left(b^{\rho}-x^{\rho}\right)^{\alpha}}\right]\right| \\
& \leq \frac{M \rho\left(b^{\rho}-a^{\rho}\right)}{2(1+p(\alpha \rho+\rho-1))^{\frac{1}{p}}}\left[\frac{1}{1-\rho s}\right]^{\frac{1}{q}} ; x \in[a, b],
\end{aligned}
$$

with $\frac{1}{p}+\frac{1}{q}=1$ where $q>1$.

Proof. Using Corollary 2.8 and then Holder's inequality, we have

$$
\begin{aligned}
& \left|f\left(x^{\rho}\right)-\frac{(\alpha \rho+\rho-1) \Gamma(\alpha)}{\rho^{1-\alpha}}\left[\frac{\rho I_{x^{-}}^{\alpha} f\left(a^{\rho}\right)}{2\left(x^{\rho}-a^{\rho}\right)^{\alpha}}+\frac{\rho^{\rho} I_{x^{+}}^{\alpha} f\left(b^{\rho}\right)}{2\left(b^{\rho}-x^{\rho}\right)^{\alpha}}\right]\right| \\
& \leq \frac{\rho\left(x^{\rho}-a^{\rho}\right)}{2} \int_{0}^{1} t^{\alpha \rho+\rho-1}\left|f^{\prime}\left(t^{\rho} x^{\rho}+\left(1-t^{\rho}\right) a^{\rho}\right)\right| d t \\
& +\frac{\rho\left(b^{\rho}-x^{\rho}\right)}{2} \int_{0}^{1} t^{\alpha \rho+\rho-1}\left|f^{\prime}\left(t^{\rho} x^{\rho}+\left(1-t^{\rho}\right) b^{\rho}\right)\right| d t \\
& \leq \frac{\rho\left(x^{\rho}-a^{\rho}\right)}{2}\left(\int_{0}^{1} t^{p(\alpha \rho+\rho-1)} d t\right)^{\frac{1}{p}} \times
\end{aligned}
$$




$$
\begin{aligned}
& \left(\int_{0}^{1}\left|f^{\prime}\left(t^{\rho} x^{\rho}+\left(1-t^{\rho}\right) a^{\rho}\right)\right|^{q} d t\right)^{\frac{1}{q}} \\
& +\frac{\rho\left(b^{\rho}-x^{\rho}\right)}{2}\left(\int_{0}^{1} t^{p(\alpha \rho+\rho-1)} d t\right)^{\frac{1}{p}} \times \\
& \left(\int_{0}^{1}\left|f^{\prime}\left(t^{\rho} x^{\rho}+\left(1-t^{\rho}\right) b^{\rho}\right)\right|^{q} d t\right)^{\frac{1}{q}} .
\end{aligned}
$$

Since $\left|f^{\prime}\right|^{q}$ is $s$-Godunova-Levin function of second kind and $\left|f^{\prime}\left(x^{\rho}\right)\right| \leq M$, we get

$$
\begin{aligned}
& \int_{0}^{1}\left|f^{\prime}\left(t^{\rho} x^{\rho}+\left(1-t^{\rho}\right) a^{\rho}\right)\right|^{q} d t \\
& \leq \int_{0}^{1}\left[\frac{1}{\left(t^{\rho}\right)^{s}}\left|f^{\prime}\left(x^{\rho}\right)\right|^{q}+\frac{1}{\left(1-t^{\rho}\right)^{s}}\left|f^{\prime}\left(a^{\rho}\right)\right|^{q}\right] d t \\
& \leq M^{q} \int_{0}^{1}\left[\frac{1}{\left(t^{\rho}\right)^{s}}+\frac{1}{\left(1-t^{\rho}\right)^{s}}\right] d t=\frac{M^{q}}{1-\rho s}
\end{aligned}
$$

similarly

$$
\int_{0}^{1}\left|f^{\prime}\left(t^{\rho} x^{\rho}+\left(1-t^{\rho}\right) b^{\rho}\right)\right|^{q} d t \leq \frac{M^{q}}{1-\rho s} .
$$

We also have

$$
\int_{0}^{1} t^{p(\alpha \rho+\rho-1)} d t=\frac{1}{1+p(\alpha \rho+\rho-1)} .
$$

Using (17), (18) and (19) in (16) we can get (15).

Corollary 2.19. In Theorem 2.18, if we take $s=0$, which means that $\left|f^{\prime}\right|$ is p-function, then (15) becomes the following inequality

$$
\begin{aligned}
& \left|f\left(x^{\rho}\right)-\frac{(\alpha \rho+\rho-1) \Gamma(\alpha)}{\rho^{1-\alpha}}\left[\frac{\rho I_{x^{-}}^{\alpha} f\left(a^{\rho}\right)}{2\left(x^{\rho}-a^{\rho}\right)^{\alpha}}+\frac{\rho^{\rho} I_{x^{+}}^{\alpha} f\left(b^{\rho}\right)}{2\left(b^{\rho}-x^{\rho}\right)^{\alpha}}\right]\right| \\
& \leq \frac{M \rho\left(b^{\rho}-a^{\rho}\right)}{2(p(\alpha \rho+\rho-1)+1)^{\frac{1}{p}}} ; x \in[a, b] .
\end{aligned}
$$

Corollary 2.20. In Theorem 2.18, if we take $s=1$, which means that $\left|f^{\prime}\right|$ is Godunova-Levin function, then (15) becomes the following inequality

$$
\begin{aligned}
& \left|f\left(x^{\rho}\right)-\frac{(\alpha \rho+\rho-1) \Gamma(\alpha)}{\rho^{1-\alpha}}\left[\frac{{ }^{\rho} I_{x^{-}}^{\alpha} f\left(a^{\rho}\right)}{2\left(x^{\rho}-a^{\rho}\right)^{\alpha}}+\frac{\rho^{\rho} I_{x^{+}}^{\alpha} f\left(b^{\rho}\right)}{2\left(b^{\rho}-x^{\rho}\right)^{\alpha}}\right]\right| \\
& \leq \frac{M \rho\left(b^{\rho}-a^{\rho}\right)}{2(p(\alpha \rho+\rho-1)+1)^{\frac{1}{p}}}\left[\frac{1}{1-\rho}\right]^{\frac{1}{q}} ; x \in[a, b] .
\end{aligned}
$$

Theorem 2.21. Let $f:\left[a^{\rho}, b^{\rho}\right] \rightarrow \mathbb{R}, a, b \geq 0, a<b$ be a differentiable function on $\left(a^{\rho}, b^{\rho}\right)$ and $f^{\prime} \in L_{1}[a, b]$. If $\left|f^{\prime}\right|^{q}$ is s-Godunova-Levin function of second kind 
and $\left|f^{\prime}\left(x^{\rho}\right)\right| \leq M, x \in[a, b], q \geq 1$, then the following inequality for Katugampola fractional integrals holds

$$
\begin{aligned}
& \left|f\left(x^{\rho}\right)-\frac{(\alpha \rho+\rho-1) \Gamma(\alpha)}{\rho^{1-\alpha}}\left[\frac{{ }^{\rho} I_{x^{-}}^{\alpha} f\left(a^{\rho}\right)}{2\left(x^{\rho}-a^{\rho}\right)^{\alpha}}+\frac{{ }^{\rho} I_{x^{+}}^{\alpha} f\left(b^{\rho}\right)}{2\left(b^{\rho}-x^{\rho}\right)^{\alpha}}\right]\right| \\
& \leq \frac{M \rho\left(b^{\rho}-a^{\rho}\right)}{2(\alpha \rho+\rho)^{1-\frac{1}{q}}}\left(\frac{1}{\rho(\alpha-s+1)}+\frac{{ }^{\rho} \Gamma(\alpha+1)^{\rho} \Gamma(1-s)}{\rho^{\rho} \Gamma(\alpha-s+2)}\right)^{\frac{1}{q}} ; x \in[a, b] .
\end{aligned}
$$

Proof. Using Corollary 2.8 and power mean inequality, we have

$$
\begin{aligned}
& \left|f\left(x^{\rho}\right)-\frac{(\alpha \rho+\rho-1) \Gamma(\alpha)}{\rho^{1-\alpha}}\left[\frac{{ }^{\rho} I_{x^{-}}^{\alpha} f\left(a^{\rho}\right)}{2\left(x^{\rho}-a^{\rho}\right)^{\alpha}}+\frac{{ }^{\rho} I_{x^{+}}^{\alpha} f\left(b^{\rho}\right)}{2\left(b^{\rho}-x^{\rho}\right)^{\alpha}}\right]\right| \\
& \leq \frac{\rho\left(x^{\rho}-a^{\rho}\right)}{2} \int_{0}^{1} t^{\alpha \rho+\rho-1}\left|f^{\prime}\left(t^{\rho} x^{\rho}+\left(1-t^{\rho}\right) a^{\rho}\right)\right| d t \\
& +\frac{\rho\left(b^{\rho}-x^{\rho}\right)}{2} \int_{0}^{1} t^{\alpha \rho+\rho-1}\left|f^{\prime}\left(t^{\rho} x^{\rho}+\left(1-t^{\rho}\right) b^{\rho}\right)\right| d t \\
& \leq \frac{\rho\left(x^{\rho}-a^{\rho}\right)}{2}\left(\int_{0}^{1} t^{\alpha \rho+\rho-1} d t\right)^{1-\frac{1}{q}} \times \\
& \left(\int_{0}^{1} t^{\alpha \rho+\rho-1}\left|f^{\prime}\left(t^{\rho} x^{\rho}+\left(1-t^{\rho}\right) a^{\rho}\right)\right|^{q} d t\right)^{\frac{1}{q}} \\
& +\frac{\rho\left(b^{\rho}-x^{\rho}\right)}{2}\left(\int_{0}^{1} t^{\alpha \rho+\rho-1} d t\right)^{1-\frac{1}{q}} \times \\
& \left(\int_{0}^{1} t^{\alpha \rho+\rho-1}\left|f^{\prime}\left(t^{\rho} x^{\rho}+\left(1-t^{\rho}\right) b^{\rho}\right)\right|^{q} d t\right)^{\frac{1}{q}} .
\end{aligned}
$$

Since $\left|f^{\prime}\right|^{q}$ is $s$-Godunova-Levin function of second kind on $\left[a^{\rho}, b^{\rho}\right]$ and $\left|f^{\prime}\left(x^{\rho}\right)\right| \leq$ $M$, we get

$$
\begin{aligned}
& \int_{0}^{1} t^{\alpha \rho+\rho-1}\left|f^{\prime}\left(t^{\rho} x^{\rho}+\left(1-t^{\rho}\right) a^{\rho}\right)\right|^{q} d t \\
& \leq \int_{0}^{1}\left[\frac{t^{\alpha \rho+\rho-1}}{\left(t^{\rho}\right)^{s}}\left|f^{\prime}\left(x^{\rho}\right)\right|^{q}+\frac{t^{\alpha \rho+\rho-1}}{\left(1-t^{\rho}\right)^{s}}\left|f^{\prime}\left(a^{\rho}\right)\right|^{q}\right] d t \\
& \leq M^{q} \int_{0}^{1}\left[\frac{t^{\alpha \rho+\rho-1}}{\left(t^{\rho}\right)^{s}}+\frac{t^{\alpha \rho+\rho-1}}{\left(1-t^{\rho}\right)^{s}}\right] d t \\
& =M^{q}\left[\frac{1}{\rho(\alpha-s+1)}+\frac{\rho^{\rho}(\alpha+1)^{\rho} \Gamma(1-s)}{\rho^{\rho} \Gamma(\alpha-s+2)}\right]
\end{aligned}
$$

similarly

$$
\int_{0}^{1} t^{\alpha \rho+\rho-1}\left|f^{\prime}\left(t^{\rho} x^{\rho}+\left(1-t^{\rho}\right) b^{\rho}\right)\right|^{q} d t
$$




$$
\leq M^{q}\left[\frac{1}{\rho(\alpha-s+1)}+\frac{{ }^{\rho} \Gamma(\alpha+1)^{\rho} \Gamma(1-s)}{\rho^{\rho} \Gamma(\alpha-s+2)}\right] .
$$

Using (22) and (23) in (21) we can attain (20).

Corollary 2.22. In Theorem 2.21, if we take $s=0$, which means that $\left|f^{\prime}\right|$ is p-function, then (20) becomes the following inequality

$$
\begin{aligned}
& \left|f\left(x^{\rho}\right)-\frac{(\alpha \rho+\rho-1) \Gamma(\alpha)}{\rho^{1-\alpha}}\left[\frac{{ }^{\rho} I_{x^{-}}^{\alpha} f\left(a^{\rho}\right)}{2\left(x^{\rho}-a^{\rho}\right)^{\alpha}}+\frac{\rho^{\rho} I_{x^{+}}^{\alpha} f\left(b^{\rho}\right)}{2\left(b^{\rho}-x^{\rho}\right)^{\alpha}}\right]\right| \\
& \leq \frac{M \rho\left(b^{\rho}-a^{\rho}\right)}{2(\rho(\alpha+1))^{1-\frac{1}{q}}}\left[\frac{2}{\rho(\alpha+1)}\right]^{\frac{1}{q}} ; x \in[a, b] .
\end{aligned}
$$

Corollary 2.23. In Theorem 2.21, if we take $s=1$, which means that $\left|f^{\prime}\right|$ is Godunova-Levin function, then (20) becomes the following inequality

$$
\begin{aligned}
& \left|f\left(x^{\rho}\right)-\frac{(\alpha \rho+\rho-1) \Gamma(\alpha)}{\rho^{1-\alpha}}\left[\frac{{ }^{\rho} I_{x^{-}}^{\alpha} f\left(a^{\rho}\right)}{2\left(x^{\rho}-a^{\rho}\right)^{\alpha}}+\frac{\rho^{\rho} I_{x^{+}}^{\alpha} f\left(b^{\rho}\right)}{2\left(b^{\rho}-x^{\rho}\right)^{\alpha}}\right]\right| \\
& \leq \frac{M \rho\left(b^{\rho}-a^{\rho}\right)}{2(\rho(\alpha+1))^{1-\frac{1}{q}}}\left[\frac{1+\alpha}{\rho \alpha}\right]^{\frac{1}{q}} ; x \in[a, b] .
\end{aligned}
$$

\section{Conclusion}

All results proved in this research paper can also be deduced for Hadamard fractional integrals just by taking limits when parameter $\rho \rightarrow 0^{+}$.

\section{Acknowledgement}

The research work of Ghulam Farid is supported by Higher Education Commission of Pakistan under NRPU 2016, Project No. 5421.

\section{Competing Interests}

The author(s) do not have any competing interests in the manuscript.

\section{REFERENCES}

1. Ostrowski, A. (1937). ber die Absolutabweichung einer differentiierbaren Funktion von ihrem Integralmittelwert. Commentarii Mathematici Helvetici, 10(1), 226-227.

2. Mitrinovic, D. S., Pecaric, J., \& Fink, A. M. (2012). Inequalities involving functions and their integrals and derivatives (Vol. 53). Springer Science \& Business Media.

3. Dragomir, S. S. (2017). Ostrowski-type inequalities for Lebesgue integral: A survey of recent results, Aust. J. Math. Anal. Appl, 14(1) 1-287.

4. Noor, M. A., Noor, K. I., \& Awan, M. U. (2014). Fractional Ostrowski inequalities for s-Godunova-Levin functions. International Journal of Analysis and Applications, 5(2), 167-173.

5. Farid, G. (2017). Some new Ostrowski type inequalities via fractional integrals. International Journal of Analysis and Applications, 14(1), 64-68.

6. Laurent, H. (1884). Sur le calcul des drives indices quelconques. Nouvelles annales de mathmatiques: journal des candidats aux coles polytechnique et normale, 3, 240-252. 
7. Hadamard, J. (1892). Essai sur l'etude des fonctions, donnees par leur developpement de Taylor. Gauthier-Villars.

8. Katugampola, U. N. (2014). A new approach to generalized fractional derivatives. Bull. Math. Anal. Appl, 6(4), 1-15.

9. Farid, G., Katugampola, U. N. \& Usman M. (2017) Ostrowski type fractional integral inequalities for mapping whose derivatives are $h$-convex via Katugampola fractional integrals. (Submitted).

10. Dragomir, S. S., Pecaric, J., \& Persson, L. E. (1995). Some inequalities of Hadamard type. Soochow J. Math, 21(3), 335-341.

11. Godunova, E. K., \& Levin, V. I. (1985). Inequalities for functions of a broad class that contains convex, monotone and some other forms of functions. Numerical mathematics and mathematical physics (Russian), 166, 138-142.

12. Noor, M. A., Noor, K. I., Awan, M. U., \& Khan, S. (2014). Fractional Hermite-Hadamard inequalities for some new classes of Godunova-Levin functions. Applied Mathematics \& Information Sciences, 8(6), 2865-2872.

13. Dragomir, S. S. (2015). Inequalities of Hermite-Hadamard type for h-convex functions on linear spaces. Proyecciones (Antofagasta), 34(4), 323-341.

\section{Ghulam Farid}

COMSATS Institute of Information Technology, Attock Campus, Pakistan.

e-mail: faridphdsms@hotmail.com, ghlmfarid@ciit-attock.edu.pk

\section{Udita N. Katugampola}

Department of Mathematics, University of Delaware, Newark, DE 19716, USA.

e-mail: uditanalin@yahoo.com

\section{Muhammad Usman}

COMSATS Institute of Information Technology, Attock, Pakistan.

e-mail: fa15-rmt-022@ciit-attock.edu.pk, usmanmani333@gmail.com 